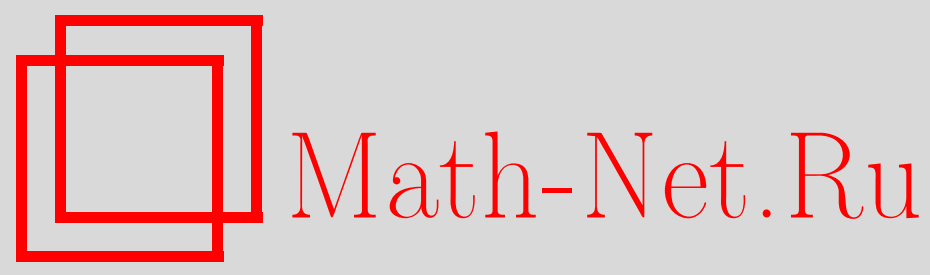

Ю. А. Пупырев, О линейной и алгебраической независимости q-дзета-значений, Матем. заметки, 2005, том 78, выпуск 4, 608-613

DOI: https://doi.org/10.4213/mzm2619

Использование Общероссийского математического портала Math-Net.Ru подразумевает, что вы прочитали и согласны с пользовательским соглашением http://www . mathnet.ru/rus/agreement

Параметры загрузки:

IP: 54.224 .187 .69

26 апреля 2023 г., $17: 30: 14$

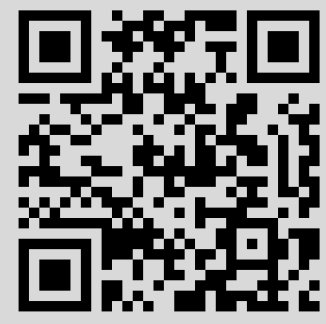




\section{О ЛИНЕЙНОЙ И АЛГЕБРАИЧЕСКОЙ НЕЗАВИСИМОСТИ $q$-ДЗЕТА-ЗНАЧЕНИЙ}

\section{Ю. А. Пупырев}

В работе получены результаты о линейной и алгебраической независимости $q$-рядов $\zeta_{q}(s)=\sum_{n=1}^{\infty} \sigma_{s-1}(n) q^{n}$, где $\sigma_{s-1}(n)=\sum_{d \mid n} d^{s-1}, s=1,2, \ldots$, над полем $\mathbb{C}(q)$.

Библиография: 3 названия.

1. Введение. Для натуральных $s=1,2, \ldots$ определим $q$-ряды

$$
\zeta_{q}(s)=\sum_{n=1}^{\infty} \sigma_{s-1}(n) q^{n}, \quad q \in \mathbb{C}, \quad|q|<1,
$$

где $\sigma_{s-1}(n)=\sum_{d \mid n} d^{s-1}$ обозначает сумму степеней делителей. Несложно проверить (например, с помощью леммы 1 далее) предельные соотношения

$$
\lim _{q \rightarrow 1}(1-q)^{s} \zeta_{q}(s)=(s-1) ! \cdot \zeta(s), \quad s=2,3, \ldots,
$$

где $\zeta(s)$ - значения дзета-функции Римана (дзета-значения). Это обстоятельство мотивирует название $q$-дзета-значения для рядов (1).

Для четных $s \geqslant 2$ ряды $E_{s}(q)=1-2 s \zeta_{q}(s) / B_{s}$, где $B_{s} \in \mathbb{Q}$ - числа Бернулли, известны как $p я д ы$ Эйзенштейна. Поэтому модулярное происхождение (относительно параметра $\tau=(\ln q) /(2 \pi i))$ функций $E_{4}, E_{6}, E_{8}, \ldots$ и теорема Малера [1] приводят к алгебраической независимости $\zeta_{q}(2), \zeta_{q}(4), \zeta_{q}(6)$ над $\mathbb{Q}[q]$, в то время как остальные четные $q$-дзета-значения являются многочленами (с коэффициентами из $\mathbb{Q}$ ) от $\zeta_{q}(4)$ и $\zeta_{q}(6)$.

В настоящей заметке мы решаем следуюшие задачи, сформулированные в [2].

ЗАДАчА 1 . Доказать, что $q$-дзета-значения $\zeta_{q}(1), \zeta_{q}(2), \zeta_{q}(3), \ldots$ как функции от $q$ линейно независимы над $\mathbb{C}(q)$.

ЗАДАчА 2. Доказать, что $q$-функциональное множество, включающее три четные $q$-дзета-значения $\zeta_{q}(2), \zeta_{q}(4), \zeta_{q}(6)$ и все нечетные $q$-дзета-значения $\zeta_{q}(1), \zeta_{q}(3)$, $\zeta_{q}(5), \ldots$, состоит из алгебраически независимых над $\mathbb{C}(q)$ функций.

Именно, мы приводим полное решение задачи 1 (теорема 1 и ее следствие) и частичное решение задачи 2 (теоремы 2-4). Отметим также, что подход к доказательству линейной и алгебраической независимости $q$-рядов, используемый в данной работе, был предложен в [3] и может применяться в других задачах.

Работа выполнена при поддержке Российского фонда фундаментальных исследований, грант № 03-01-00359. 


\section{2. Вспомогательные утверждения.}

ЛЕмма 1. Для $\zeta_{q}(s), s=1,2, \ldots$, имеем представление

$$
\zeta_{q}(s)=\sum_{n=1}^{\infty} \frac{q^{n} \rho_{s}\left(q^{n}\right)}{\left(1-q^{n}\right)^{s}}
$$

где $\rho_{s}-$ многочлен со следующими свойствами:

1) все его коэффичиенты положсительны;

2) при $s>1$ его степень равна $s-2$, при $s=1$ степень равна 0 ;

3) $\rho_{s}(1)=(s-1) !$

ДокАЗАТЕльСтво. Докажем сначала представление (2):

$$
\begin{aligned}
\zeta_{q}(s) & =\sum_{n=1}^{\infty} \sigma_{s-1}(n) q^{n}=\sum_{\nu=1}^{\infty} \nu^{s-1} \sum_{n=1}^{\infty} q^{\nu n}=\left.\sum_{n=1}^{\infty}\left(\sum_{\nu=1}^{\infty} \nu^{s-1} x^{\nu}\right)\right|_{x=q^{n}} \\
& =\left.\sum_{n=1}^{\infty}\left(\left(x \frac{d}{d x}\right)^{s-1} \frac{x}{1-x}\right)\right|_{x=q^{n}}=\left.\sum_{n=1}^{\infty} \frac{x \rho_{s}(x)}{(1-x)^{s}}\right|_{x=q^{n}}=\sum_{n=1}^{\infty} \frac{q^{n} \rho_{s}\left(q^{n}\right)}{\left(1-q^{n}\right)^{s}} .
\end{aligned}
$$

Теперь воспользуемся тем, что $\rho_{1} \equiv 1$, и по индукции проверим указанные вьше свойства многочлена $\rho_{s}$. Пусть $\gamma_{s}(x)=x \rho_{s}(x)$ - многочлен степени не вьше $s$ с неотрицательными коэффициентами. Тогда

$$
\frac{\gamma_{s+1}(x)}{(1-x)^{s+1}}=\left(x \frac{d}{d x}\right) \frac{\gamma_{s}(x)}{(1-x)^{s}}=x \frac{\gamma_{s}^{\prime}(x)(1-x)+s \gamma_{s}(x)}{(1-x)^{s+1}}=x \frac{\left(s \gamma_{s}(x)-x \gamma_{s}^{\prime}(x)\right)+\gamma_{s}^{\prime}(x)}{(1-x)^{s+1}}
$$

Заметим, что коэффициенты $\gamma_{s}^{\prime}$ получаются из коэффициентов $\gamma_{s}$ умножением на числа, не превосходяшие $s$. Поэтому, учитьвая, что $\gamma_{s+1}(x)=x \rho_{s+1}(x)$, убеждаемся в справедливости свойства 1) и получаем рекуррентное соотношение для $\rho_{s}$ :

$$
\rho_{s+1}(x)=(1+(s-1) x) \rho_{s}(x)+x(1-x) \rho_{s}^{\prime}(x),
$$

откуда следуют свойства 2) и 3). Лемма доказана.

Напомним, что запись $f(x) \asymp g(x)$ при $x \rightarrow a$ означает, что с некоторыми постоянными $C_{1}, C_{2}>0$ выполнено

$$
C_{1}|g(x)| \leqslant|f(x)| \leqslant C_{2}|g(x)| \quad \text { при } x \rightarrow a .
$$

Лемма 2. Пусть $\theta$ - некоторый корень из единицы и $q=r \theta$, где $0<r<1$. Тогда

1) $\partial$ ภя $s>1$

$$
\zeta_{q}(s) \asymp \frac{1}{(1-r)^{s}} \quad \text { npu } \quad r \rightarrow 1
$$

2) для $s=1$

$$
\zeta_{q}(1) \asymp-\frac{\ln (1-r)}{1-r} \quad \text { npu } \quad r \rightarrow 1 .
$$


ДокАЗАТЕЛЬСтвО. Пусть $\theta$ - первообразньй корень степени $p$ из единищы, а число $s$ фиксировано. Запишем ряд из $(2)$ в виде $\zeta_{q}(s)=\Sigma_{1}+\Sigma_{2}$, где

$$
\Sigma_{1}=\sum_{n \geqslant 1, p \mid n} \frac{q^{n} \rho_{s}\left(q^{n}\right)}{\left(1-q^{n}\right)^{s}}, \quad \Sigma_{2}=\sum_{n \geqslant 1, p \nmid n} \frac{q^{n} \rho_{s}\left(q^{n}\right)}{\left(1-q^{n}\right)^{s}} .
$$

Если $p \nmid n$, то $\left|1-q^{n}\right|=\left|1-r^{n} \theta^{n}\right| \geqslant c_{1}>0$, так как множество значений $q^{n}=r^{n} \theta^{n}$ расположено на радиальных лучах, не проходящих через 1 , а значит, разность $\left|1-q^{n}\right|$ оценивается снизу константой, равной расстоянию от 1 до данного множества. Для $p \geqslant 4$ это расстояние равно $\sin (2 \pi / p)$, если же $p<4$, то оно равно 1 . Итак, постоянная $c_{1}$ зависит только от $p$. И в дальнейшем символы $c_{2}, c_{3}, \ldots$ будут обозначать положительные величины, не зависящие от $r$.

В области $|q|<1$ значения $\rho_{s}\left(q^{n}\right)$ ограничены суммой модулей коэффищиентов многочлена, которая в силу их положительности и леммы 1 равна $(s-1) !$. Пользуясь данным фактом и установленным вьше неравенством, получаем

$$
\left|\Sigma_{2}\right| \leqslant c_{1}^{-s}(s-1) ! \sum_{n \geqslant 1, p \nmid n} r^{n} \leqslant c_{1}^{-s}(s-1) ! \sum_{n=0}^{\infty} r^{n} \leqslant \frac{c_{2}}{1-r} .
$$

Заметим, что это верно для любого $s \in \mathbb{N}$.

Далее отдельно рассмотрим случаи $s>1$ и $s=1$.

Пусть $s>1$. Из леммы 1 также следует, что многочлен $\rho_{s}$ является положительным и неубывающим на вещественной полуоси $[0,+\infty)$, а его свободный член не равен нулю, так что значения многочлена ограничены снизу некоторой константой $c_{3}>0$. Поэтому при $r>1 / 2$ справедлива следующая оценка:

$$
\Sigma_{1}=\sum_{l=1}^{\infty} \frac{r^{p l} \rho_{s}\left(r^{p l}\right)}{\left(1-r^{p l}\right)^{s}} \geqslant \frac{c_{3} r^{p}}{\left(1-r^{p}\right)^{s}} \geqslant \frac{c_{4}}{(1-r)^{s}} .
$$

Для оценки сверху разобьем множество возможных значений индекса суммирования $l$ на непересекающиеся подмножества $S_{N}$ так, что $l \in S_{N}$, если $N=\left[1+r+\cdots+r^{p l-1}\right]$, где [· - целая часть числа. Тогда

$$
\begin{aligned}
\Sigma_{1} & =\sum_{l=1}^{\infty} \frac{r^{p l} \rho_{s}\left(r^{p l}\right)}{\left(1-r^{p l}\right)^{s}} \leqslant(s-1) ! \frac{1}{(1-r)^{s}} \sum_{l=1}^{\infty} \frac{r^{p l}}{\left(1+r+\cdots+r^{p l-1}\right)^{s}} \\
& \leqslant(s-1) ! \frac{1}{(1-r)^{s}} \sum_{N=1}^{[1 /(1-r)]} \frac{1}{N^{s}} \sum_{l \in S_{N}} r^{p l} .
\end{aligned}
$$

Согласно построению множеств $S_{N}$ вьполнено

$0<\sum_{l \in S_{N}} r^{p l}=r^{p l_{*}}+r^{p\left(l_{*}+1\right)}+\cdots+r^{p\left(l^{*}-1\right)+r p l^{*}} \leqslant r^{p l_{*}}+r^{p l_{*}+1}+\cdots+r^{p l^{*}-1}+1 \leqslant 2$,

где $l_{*}=\min _{l \in S_{N}}\{l\}$, a $l^{*}=\max _{l \in S_{N}}\{l\}$, откуда

$$
\Sigma_{1} \leqslant 2(s-1) ! \frac{1}{(1-r)^{s}} \sum_{N=1}^{\infty} \frac{1}{N^{s}} \leqslant \frac{c_{5}}{(1-r)^{s}} .
$$


Таким образом, первая часть леммы доказана.

Пусть теперь $s=1$. Тогда

$$
\Sigma_{1}=\sum_{l=1}^{\infty} \frac{r^{p l}}{1-r^{p l}}=\frac{1}{1-r} \sum_{l=1}^{\infty} \frac{r^{p l}}{1+r+\cdots+r^{p l-1}}=\frac{1}{1-r} \psi(r)
$$

Так как $1+r+\cdots+r^{p l-1} \leqslant p l$, получаем

$$
\psi(r) \geqslant c_{6} \sum_{l=1}^{\infty} \frac{r^{p l}}{p l}=-c_{6} \frac{1}{p} \ln \left(1-r^{p}\right) \geqslant-c_{7} \ln (1-r),
$$

откуда

$$
\Sigma_{1} \geqslant-\frac{c_{7} \ln (1-r)}{1-r}
$$

Теперь оценим $\Sigma_{1}$ сверху. Так же, как и в доказательстве оценки $(3)$, получаем

$$
\begin{aligned}
\Sigma_{1} & =\sum_{l=1}^{\infty} \frac{r^{p l}}{1-r^{p l}} \leqslant 2 \frac{1}{1-r}\left(1+\frac{1}{2}+\cdots+\frac{1}{[1 /(1-r)]}\right) \\
& \leqslant 2 \frac{1}{1-r}\left(1+\int_{1}^{1 /(1-r)} \frac{d x}{x}\right) \leqslant c_{8} \frac{-\ln (1-r)}{1-r}
\end{aligned}
$$

что завершает доказательство леммы.

3. Основные результаты. Пусть даны ненулевые многочлены $P_{i}(q) \in \mathbb{C}[q], i=$ $1, \ldots, k$, со степенями $d_{i}$ соответственно. Определим линейную комбинацию

$$
P(q)=P\left(s_{1}, \ldots, s_{k} ; q\right)=\sum_{i=1}^{k} P_{i}(q) \zeta_{q}\left(s_{i}\right)
$$

где $s_{1}>\cdots>s_{k}$. Через $\Omega \subset \mathbb{C}$ обозначим мультипликативную группу всех корней из единицы.

Теорема 1. Начиная с некоторого $p_{0}$, зависящего от $s_{1}, s_{2}, \ldots, s_{k}$ и многочлена $P_{1}(q)$, каждый первообразный корень $p$-й степени из единицы, $p \geqslant p_{0}$, является особой точкой функиии $P(q)$. В частности, мнохсество всех особых точек функuии (4) всюду плотно на окружности $|q|=1$, а функция $P(q)$ трансцендентна.

ДокАЗАТЕЛЬСТво. Возьмем число $\theta \in \Omega$, не являющееся корнем многочлена $P_{1}$, и пусть $q=r \theta$. Представим многочлены $P_{i}, i=1, \ldots, k$, в виде

$$
P_{i}(q)=\sum_{m=0}^{d_{i}} a_{m, i}(\theta-q)^{m}
$$


Тогда

$$
\begin{aligned}
P(q) & =\sum_{i=1}^{k} \sum_{m=0}^{d_{i}} a_{m, i}(\theta-q)^{m} \zeta_{q}\left(s_{i}\right) \\
& =a_{0,1} \zeta_{q}\left(s_{1}\right)+\sum_{i=2}^{k} a_{0, i} \zeta_{q}\left(s_{i}\right)+\theta(1-r) \sum_{i=1}^{k} \sum_{m=1}^{d_{i}} a_{m, i} \theta^{m-1}(1-r)^{m-1} \zeta_{q}\left(s_{i}\right),
\end{aligned}
$$

откуда в соответствии с леммой 2 при $r \rightarrow 1$

$$
\begin{aligned}
& P(q) \asymp \frac{1}{(1-r)^{s_{1}}}, \quad \text { если } s_{1} \geqslant 2, \\
& P(q) \asymp-\frac{\ln (1-r)}{1-r}, \quad \text { если } s_{1}=1 .
\end{aligned}
$$

Поэтому $\theta$ - особая точка функции (4).

Для завершения доказательства остается заметить, что алгебраическая функция имеет особенности лишь в конечном числе точек.

СлЕДСТВИЕ. Функции $\zeta_{q}(s), s \geqslant 1$, линейно независимы над $\mathbb{C}(q)$.

ЗАмечАнИЕ. Вместо многочленов $P_{i}$ в $(4)$ можно рассматривать целые функции (в этом случае ряды в (5) бесконечны). Тогда результат теоремы 1 можно усилить, заменив поле $\mathbb{C}(q)$ на поле мероморфных функций.

ТЕОРема 2. При любом $s>1$ функиии $\zeta_{q}(1)$ u $\zeta_{q}(s)$ алгебраически независимы над $\mathbb{C}(q)$.

ДокАЗАТЕЛЬСтво. Пусть от противного существует многочлен $P\left(x_{0}, x_{1}\right)$ с коэффициентами из $\mathbb{C}[q]$ такой, что $P\left(\zeta_{q}(1), \zeta_{q}(s)\right) \equiv 0$. Запишем его в виде

$$
P\left(x_{0}, x_{1}\right)=\sum_{i} P_{i}(q) x_{0}^{l_{0, i}} x_{1}^{l_{1, i}}
$$

где суммирование распространяется на все ненулевые коэффициенты $P_{i}(q)$ многочлена $P\left(x_{0}, x_{1}\right)$.

Выберем число $\theta \in \Omega$, не являющееся корнем ни одного из $P_{i}(q)$, и пусть $q=r \theta$. Рассмотрим те $i$, для которых значение суммы $l_{1, i} s+l_{0, i}$ максимально, и выберем из них то, для которого максимально $l_{0, i}$. Пусть это $l_{0, i}=l_{0}$, а соответствующее $l_{1, i}=l_{1}$. Тогда, проводя те же рассуждения, что и в доказательстве теоремы 1, получаем

$$
P\left(\zeta_{q}(1), \zeta_{q}(s)\right) \asymp(-\ln (1-r))^{l_{0}} \frac{1}{(1-r)^{l_{1} s+l_{0}}} \quad \text { при } \quad r \rightarrow 1 .
$$

Это противоречит предположению $P\left(\zeta_{q}(1), \zeta_{q}(s)\right) \equiv 0$, откуда и следует утверждение теоремы.

ТЕОремА 3. Пусть для некоторого набора $s_{1}, s_{2}, \ldots, s_{k} \in \mathbb{N}$ чисел таких, что $s_{i}>1, \quad i=1, \ldots, k$, функиии $\zeta_{q}\left(s_{1}\right), \zeta_{q}\left(s_{2}\right), \ldots, \zeta_{q}\left(s_{k}\right)$ алгебраически независимы над $\mathbb{C}(q)$. Тогда функиии $\zeta_{q}(1)$ и $\zeta_{q}\left(s_{1}\right), \zeta_{q}\left(s_{2}\right), \ldots, \zeta_{q}\left(s_{k}\right)$ также алгебраически независимы над $\mathbb{C}(q)$. 
ДокАЗАТЕЛЬСТвО. Произвольньй многочлен $P\left(x_{0}, x_{1}, \ldots, x_{k}\right)$ с коэффициентами из $\mathbb{C}[q]$ можно представить в виде

$$
P\left(x_{0}, x_{1}, \ldots, x_{k}\right)=\sum_{i} P_{i}(q) x_{0}^{l_{0, i}} x_{1}^{l_{1, i}} \cdots x_{k}^{l_{k, i}}
$$

где $P_{i}(q) \in \mathbb{C}[q]$ - все ненулевые коэффициенты $P\left(x_{0}, x_{1}, \ldots, x_{k}\right)$.

Аналогично доказательству теоремы 2 выберем число $\theta \in \Omega$, не являющееся корнем ни одного из многочленов $P_{i}$, и пусть $q=r \theta$. Из леммы 2 для функций $\zeta_{q}(s), s>1$, имеем

$$
\zeta_{q}(s)=\frac{1}{(1-r)^{s}} \chi_{s}(r)
$$

где $\chi_{s}(r)$ - ограниченные на отрезке $[0,1]$ функции. Теперь представим многочлен $(6)$ в виде

$$
P\left(x_{0}, x_{1}, \ldots, x_{k}\right)=\sum_{l} x_{0}^{l} \sum_{i \in I(l)} P_{i}(q) x_{1}^{l_{1, i}} \cdots x_{k}^{l_{k, i}},
$$

где $I(l)$ - множество значений индекса $i$, для которых $l_{0, i}=l$. Из алгебраической независимости функций $\zeta_{q}\left(s_{1}\right), \zeta_{q}\left(s_{2}\right), \ldots, \zeta_{q}\left(s_{k}\right)$ и представления (7) для любого $l$ получаем

$$
\sum_{i \in I(l)} P_{i}(q) \zeta_{q}\left(s_{1}\right)^{l_{1, i}} \cdots \zeta_{q}\left(s_{k}\right)^{l_{k, i}} \asymp \frac{1}{(1-r)^{m(l)}} \quad \text { при } \quad r \rightarrow 1,
$$

где $m(l)$ - целое неотрицательное число. Рассмотрим те $l$, для которых значение суммы $m(l)+l$ максимально, и выберем из них максимальное $l=l^{*}$. Тогда

$$
P\left(\zeta_{q}(1), \zeta_{q}\left(s_{1}\right), \ldots, \zeta_{q}\left(s_{k}\right)\right) \asymp(-\ln (1-r))^{l^{*}} \frac{1}{(1-r)^{m\left(l^{*}\right)+l^{*}}} \quad \text { при } \quad r \rightarrow 1
$$

и, в частности, выражение слева отлично от нуля. Теорема доказана.

ТЕОрема 4. Функиии $\zeta_{q}(1)$ u $\zeta_{q}(2), \zeta_{q}(4), \zeta_{q}(6)$ алгебраически независимы над $\mathbb{C}(q)$.

ДокАЗАТЕльство следует из алгебраической независимости $\zeta_{q}(2), \zeta_{q}(4), \zeta_{q}(6)($ см. введение), доказанной в [1], и теоремы 3.

\section{СПИСОК ЦИТИРОВАННОЙ ЛИТЕРАТУРЫ}

[1] Mahler K. On algebraic differential equations satisfied by automorphic functions // J. Austral. Math. Soc. 1969. V. 10. P. 445-450.

[2] Зудилин В. В. О диофантовых задачах для $q$-дзета-значений // Матем. заметки. 2002. Т. 72. №6. С. 936-940.

[3] Нестеренко Ю. В. О трансцендентности некоторых функций. Рукопись (2003).

Московский государственный университет им. М.В. Ломоносова

Поступило

04.04 .2005 